\title{
Accuracy of c-KIT in lung cancer prognosis; a systematic review protocol" instead of c-KIT Expression in Lung Cancer Prognostic Evaluation - a Systematic Review Protocol
}

\author{
Raheleh Roudi ${ }^{1}$, Elham Kalantari ${ }^{1}$, Abbas Keshtkar ${ }^{2}$, Zahra Madjd ${ }^{1,3 *}$
}

\begin{abstract}
Background: Extensive efforts have been made to investigate c-KIT expression in lung cancer specimens and its correlation with clinical outcomes, but the issue remains unresolved. Thus, this study will be conducted to clarify the prognostic value of c-KIT expression in lung cancer patients. Materials and Methods: We will search Pubmed, SCOPUS, and ISI web of sciences with no restriction of language. Studies with any design (except case reports or case series) evaluating correlations of c-KIT expression with survival or outcome in patients with lung cancer will be included. The outcome measures will include all types of survival indexes, including overall survival rate and disease free survival using Kaplan-Meier analysis and hazard ratios. Study selection and data extraction will be performed by two independent researchers. Quality assessment (assessment of risk of bias) and data synthesis will be implemented using Stata software version 11.1. Results: No ethical issues are predicted. These findings will be published in a peer-reviewed journal and presented at national and international conferences. Conclusions: This systematic review protocol is registered in the PROSPERO International Prospective Register of Systematic Reviews, registration number $=$ CRD42015023391.
\end{abstract}

Keywords: c-KIT - CD117 - Lung cancer - Systematic review

Asian Pac J Cancer Prev, 17 (2), 863-866

\section{Introduction}

Lung cancer is the most common cancer-related death in both men and women in all over the world (Siegel et al., 2013; Siegel et al., 2014). For diagnostic and therapeutic purposes, two main groups of lung cancer are distinguished: small cell lung cancer (SCLC) and nonsmall cell lung cancer (NSCLC) (Ettinger et al., 2012; Kalemkerian et al., 2013). NSCLC that represents nearly $80 \%$ to $85 \%$ of all lung cancer, is a highly heterogeneous group including: squamous cell carcinoma (SCC), adenocarcinoma (ADC), and large cell carcinoma (LCC) (Ettinger et al., 2012). SCLC, which accounts for 15$20 \%$ of all lung cancers, exhibits a highly aggressive and metastatic potential that NSCLC (Kalemkerian et al., 2013). The 5-year survival rates in all stages of lung cancer remains only $15 \%$ and more than half of the patients at diagnosis $(54 \%)$ have advanced disease namely distant stage or metastasis (Ettinger et al., 2012; Siegel et al., 2013; Siegel et al., 2014). These catastrophic mortalities and morbidities, as well as, short-term survival of patients' high indicated that there is an urgent necessity for finding reliable prognostic marker(s) and new agent(s) for targeted therapy in lung cancer patients.

A wide spectrum of biomarkers has been tested for their prognostic and predictive values in lung cancer and some have used in clinical applications, including K-RAS, EGF-R, and thyroid transcription factor-1 (TTF1) (Mascaux et al., 2005; Berghmans et al., 2006; Nakamura et al., 2006; Berghmans et al., 2011; Gazdar and Schiller 2011). In the two last decades, proto-oncogene c-KIT has been great of attention for a prognostic role in several malignant tumors, especially lung cancer (Grossi et al., 2010; Medinger et al., 2010; Herpel et al., 2011; Lu et al., 2012; Xiao et al., 2014; Matsumura et al., 2015). The proto-oncogene c-KIT, also known as CD117, encodes a transmembrane protein that belongs to tyrosine kinase receptor type III (Chabot et al., 1988; Ashman, 1999). c-KIT binds its ligand, that's called stem cell factor (SCF), and plays important roles in cell survival, proliferation, and differentiation (Edling and Hallberg 2007).

The correlation between c-KIT expressions and clinicopathlogical parameters including disease free survival (DFS) or overall survival (OS) was explored in lung cancer patients, but there is no consensus concerning the prognostic role of c-KIT these patients (Mascaux

${ }^{1}$ Oncopathology Research Center, Iran University of Medical Sciences, ${ }^{2}$ Osteoporosis Research Center (ORC), Endocrinology and Metabolism Clinical Sciences Research Institute (ECSI), Tehran University of Medical Sciences, ${ }^{3}$ Department of Molecular Medicine, Faculty of Advanced Technologies in Medicine, Iran University of Medical Sciences, Tehran, Iran *For correspondence: Zahra.madjd@yahoo.com 
et al., 2005; Berghmans et al., 2006; Nakamura et al., 2006; Berghmans et al., 2011; Gazdar and Schiller 2011). Although a more recent attempt to investigate the prognostic value of c-KIT expression in cancer patients (Zhao et al., 2014), this systematic review, for the first time, will conduct to evaluate the prognostic accuracy of c-KIT in the prognosis of patients with lung cancer using comprehensive search of several databases.

\section{Objectives}

The primary objective of the current systematic review is to assess the prognostic accuracy of c-KIT in lung cancer patients using survival measure. The secondary objectives will be to determine prognostic accuracy of c-KIT in different subtypes of lung cancer, including SCC, ADC, and LCC in NSCLC group and SCLC group, as well as, determination of the heterogeneity and its potential source.

\section{Methods}

The protocol of this systematic review has been registered on PROSPERO website (www.crd. york.ac.uk/PROSPERO) (PROSPERO registration number=CRD42015023391). This systematic review protocol has been developed based on the Preferred Reporting Items for Systematic Reviews and MetaAnalyses (PRISMA) statement guidelines (Liberati et al., 2009).

\section{Criteria for considering studies for this systematic review.}

Types of studies; All observational studies (casecontrol, cross sectional, and cohort) have been evaluated the association between c-KIT expression and the clinical prognosis of lung cancer will include in the current study.

\section{Participants}

The studies will include in this systematic review that evaluated the association between c-KIT expression and the clinical prognosis or survival outcome (disease free survival or overall survival) of patients with lung cancer. Both of gender (female and male), participants with any limitation of age, patients with no preoperatively treatment of all histological grades (well, moderate, and poor) and stages (I, II, III, and IV) will be included in the current systematic review. In addition lymph node involvement and metastasis data will be recorded without any limitation.

\section{Index Test}

In the current study, c-KIT expression will define by immunohistochemistry (IHC) or tissue microarray (TMA) with all scoring systems, including intensity of staining, percentage of positive cells, and H-score (McCarty et al., 1985). Evaluation of c-KIT using flow cytometry or molecular-based approaches such as polymerase chain reaction (PCR) were excluded from the current systematic review.

\section{Gold standard}

All types of survival indexes, including survival rate, disease free survival, Kaplan-Meier analysis, and hazard ratio will be included in the current systematic review.

\section{Target Condition}

Prognosis of lung cancer patients will be target condition.

\section{Outcomes}

The our primary outcomes will be all types of survival indexes, including survival rate, disease free survival, Kaplan-Meier analysis, and hazard ratio. In addition, the secondary outcomes the current systematic review will be to determine prognostic accuracy of c-KIT in different subtypes of lung cancer, including SCC, ADC, and LCC in NSCLC group and SCLC group, as well as, determination of the heterogeneity and its potential source.

\section{Search Methods for Identification of Studies}

The electronic databases Medline, SCOPUS, and ISI web of sciences will be searched to end of Jun 2015.

\section{Searching Strategies of the Medline Database}

MeSH tags were found in the MEDLINE subset of PubMed. Then, we used the syntax number 3 for selecting the relevant clinical trials.

i). c-KIT; ii). CD117; iii). p145; iv). Stem cell factor; v). c- KIT OR CD117 OR p145 OR stem cell factor; vi). c- KIT [tiab] OR CD117 [tiab] OR p145 [tiab] OR stem cell factor [tiab]; vii). (c-KIT [tiab] OR CD117 [tiab] OR p145 [tiab] OR stem cell factor [tiab]) AND (lung or pulmonary) (Table 1).

\section{Searching Strategies of the SCOPUS}

The search terms and syntax for SCOPUS will be as follow (Table 2): i). (TITLE-ABS-KEY (c-KIT) OR

Table 1. Search Syntax in Pubmed

\begin{tabular}{cl}
\hline Syntax no. & \multicolumn{1}{c}{ Syntax } \\
\hline 1 & c-KIT OR CD117 OR p145 OR stem cell factor \\
2 & c- KIT [tiab] OR CD117 [tiab] OR p145 [tiab] OR stem cell factor [tiab] \\
3 & (c- KIT [tiab] OR CD117 [tiab] OR p145 [tiab] OR stem cell factor [tiab]) AND (lung or pulmonary) \\
\hline
\end{tabular}




\begin{tabular}{|c|c|}
\hline Syntax no. & Syntax \\
\hline 1 & $\begin{array}{l}\text { (TITLE-ABS-KEY (c-KIT) OR TITLE-ABS-KEY (CD117) OR TITLE-ABS-KEY (p145) OR TITLE-ABS-KEY } \\
\text { (stem cell factor) }\end{array}$ \\
\hline 2 & $\begin{array}{l}\text { TITLE-ABS-KEY (c-KIT) OR TITLE-ABS-KEY (CD117) OR TITLE-ABS-KEY (p145) OR TITLE-ABS-KEY } \\
\text { (stem cell factor) AND TITLE-ABS-KEY (lung) OR TITLE-ABS-KEY (pulmonary) }\end{array}$ \\
\hline ( & $\begin{array}{l}\text { (TITLE-ABS-KEY (c-KIT) OR TITLE-ABS-KEY (CD117) OR TITLE-ABS-KEY (p145) OR TITLE-ABS-KEY } \\
\text { (stem cell factor) AND TITLE-ABS-KEY (lung) OR TITLE-ABS-KEY (pulmonary) AND TITLE-ABS-KEY (cancer) } \\
\text { OR TITLE-ABS-KEY (tumor) OR TITLE-ABS-KEY (carcinoma) OR TITLE-ABS-KEY (neoplasm) } \\
\text { OR TITLE-ABS-KEY (neoplasia) }\end{array}$ \\
\hline ( & $\begin{array}{l}\text { (TITLE-ABS-KEY (c-kit) OR TITLE-ABS-KEY (cd117) OR TITLE-ABS-KEY (p145) OR TITLE-ABS-KEY } \\
\text { (stem cell factor) AND TITLE-ABS-KEY (lung) OR TITLE-ABS-KEY (pulmonary) AND TITLE-ABS-KEY (cancer) } \\
\text { OR TITLE-ABS-KEY (tumor) O TITLE-ABS-KEY (carcinoma) OR TITLE-ABS-KEY (neoplasm) OR TITLE-ABS-KEY } \\
\text { (neoplasia) AND TITLE-ABS-KEY (prognosis) OR TITLE-ABS-KEY (disease free survival) OR TITLE-ABS-KEY } \\
\text { (outcome) OR TITLE-ABS-KEY (prognostic)) }\end{array}$ \\
\hline
\end{tabular}

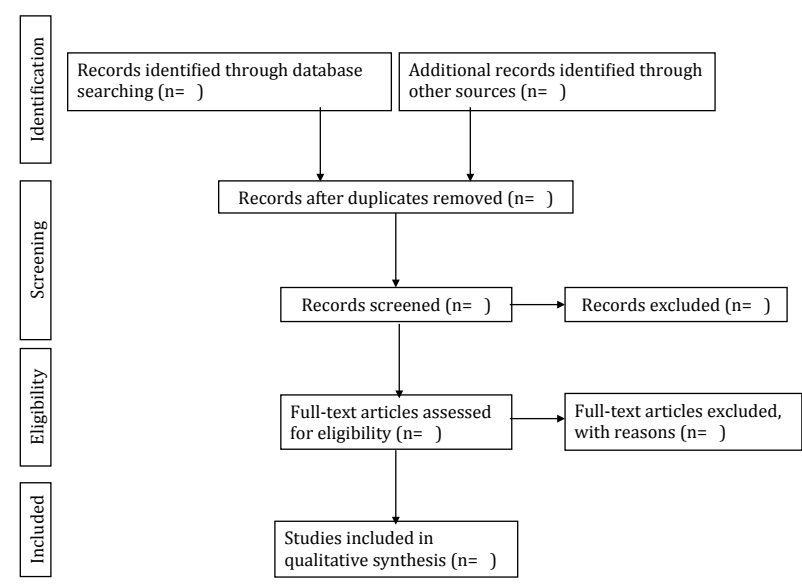

Figure 1. Flow Diagram of the Study Selection Process Based on PRISMA Guidelines

TITLE-ABS-KEY (CD117) OR TITLE-ABS-KEY (p145) OR TITLE-ABS-KEY (stem cell factor); ii). TITLE-ABSKEY (c-KIT) OR TITLE-ABS-KEY (CD117) OR TITLEABS-KEY (p145) OR TITLE-ABS-KEY (stem cell factor) AND TITLE-ABS-KEY (lung) OR TITLE-ABSKEY (pulmonary); iii). (TITLE-ABS-KEY (c-KIT) OR TITLE-ABS-KEY (CD117) OR TITLE-ABS-KEY (p145) OR TITLE-ABS-KEY (stem cell factor) AND TITLEABS-KEY (lung) OR TITLE-ABS-KEY (pulmonary) AND TITLE-ABS-KEY (cancer) OR TITLE-ABS-KEY (tumor) OR TITLE-ABS-KEY (carcinoma) OR TITLEABS-KEY (neoplasm) OR TITLE-ABS-KEY (neoplasia); iv). (TITLE-ABS-KEY (c-kit) OR TITLE-ABS-KEY (cd117) OR TITLE-ABS-KEY (p145) OR TITLE-ABSKEY (stem cell factor) AND TITLE-ABS-KEY (lung) OR TITLE-ABS-KEY (pulmonary) AND TITLE-ABS-KEY (cancer) OR TITLE-ABS-KEY (tumor) OR TITLE-ABSKEY (carcinoma) OR TITLE-ABS-KEY (neoplasm) OR TITLE-ABS-KEY (neoplasia) AND TITLE-ABS-KEY (prognosis) OR TITLE-ABS-KEY (disease free survival) OR TITLE-ABS-KEY (outcome) OR TITLE-ABS-KEY (prognostic)

\section{Data Collection}

Studies identified by the final syntax in electronic searches screened with the two authors (RR and EK) will independently screen titles and abstracts to identify potentially relevant studies. Disagreements will be resolved by discussion between the two authors (RR and EK). After the screening, we will retrieve full texts of potentially eligible studies to assess whether the individual studies fulfill the inclusion criteria. Two authors (RR and EK) will independently extract data from the selected original articles. If there are any discrepancies, it will be resolved by consensus between the two authors. As shown in figure 1, the study selection is performed based on the PRISMA flow diagram.

\section{Data Analysis}

Statistical analysis of data will be performed using Stata software version 11.1 (StataCorp LP, College Station, TX, USA). The association of c-KIT expression and clinicopathological features as well as DFS or OS will be assessed using forest plot. Study heterogeneity will be measured using the $\mathrm{Q}$ test and $\mathrm{I}^{2}$ test. For evaluating source of heterogeneity, sub-group analysis and metaregression will be used. The potential for publication bias was assessed using funnel plot, the Begg rank correlation method, and the Egger weighted regression method. A p-value of $\leq 0.05$ will be considered to be statistically significant.

\section{References}

Ashman LK (1999). The biology of stem cell factor and its receptor C-kit. Int J Biochem Cell Biol, 31, 1037-51.

Berghmans T, Mascaux C, Martin B, et al (2006). Prognostic role of thyroid transcription factor-1 in stage III non-small cell lung cancer. Lung Cancer, 52, 219-24.

Berghmans T, Paesmans M, Sculier J P (2011). Prognostic factors in stage III non-small cell lung cancer: a review of conventional, metabolic and new biological variables. Ther Adv Med Oncol, 3, 127-38.

Chabot B, Stephenson D A, Chapman V M, et al (1988). The proto-oncogene c-kit encoding a transmembrane tyrosine kinase receptor maps to the mouse W locus. Nature, $\mathbf{3 3 5}$, 88-9.

Edling C E, Hallberg B (2007). c-Kit-a hematopoietic cell essential receptor tyrosine kinase. Int J Biochem Cell Biol, 39, 1995-8.

Ettinger D S, Akerley W, Borghaei H, et al (2012). Non-small 
cell lung cancer. J Natl Compr Canc Netw, 10, 1236-71.

Gazdar A F , Schiller J H (2011). Predictive and prognostic factors for non-small cell lung cancer-potholes in the road to the promised land. J Natl Cancer Inst, 103, 1810-1.

Grossi F , Spizzo R, Bordo D, et al (2010). Prognostic stratification of stage IIIA pN2 non-small cell lung cancer by hierarchical clustering analysis of tissue microarray immunostaining data: an alpe adria thoracic oncology multidisciplinary group study (ATOM 014). J Thorac Oncol, 5, 1354-60.

Herpel E, Jensen K, Muley T, et al (2011). The cancer stem cell antigens CD133, BCRP1/ABCG2 and CD117/c-KIT are not associated with prognosis in resected early-stage non-small cell lung cancer. Anticancer Res, 31, 4491-500.

Kalemkerian GP, Akerley W, Bogner P, et al (2013). Small cell lung cancer. J Natl Compr Canc Netw, 11, 78-98.

Liberati A, Altman D G, Tetzlaff J, et al (2009). The PRISMA statement for reporting systematic reviews and metaanalyses of studies that evaluate health care interventions: explanation and elaboration. Ann Intern Med, 151, 65-94.

Lu H Y, Zhang G, Cheng Q Y, et al (2012). Expression and mutation of the c-kit gene and correlation with prognosis of small cell lung cancer. Oncol Lett, 4, 89-93.

Mascaux C, Iannino N, Martin B, et al (2005). The role of RAS oncogene in survival of patients with lung cancer: a systematic review of the literature with meta-analysis. $\mathrm{Br} J$ Cancer, 92, 131-9.

Matsumura Y , Umemura S, Ishii G, et al (2015). Expression profiling of receptor tyrosine kinases in high-grade neuroendocrine carcinoma of the lung: a comparative analysis with adenocarcinoma and squamous cell carcinoma. $J$ Cancer Res Clin Oncol, 1-12.

McCarty Jr K, Miller L, Cox E, et al (1985). Estrogen receptor analyses. correlation of biochemical and immunohistochemical methods using monoclonal antireceptor antibodies. Arch Pathol Lab Med, 109, 716-21.

Medinger M, Kleinschmidt M, Mross K, et al (2010). c-kit (CD117) expression in human tumors and its prognostic value: an immunohistochemical analysis. Pathol Oncol Res, 16, 295-301.

Nakamura H, Kawasaki N, Taguchi M, et al (2006). Survival impact of epidermal growth factor receptor overexpression in patients with non-small cell lung cancer: a meta-analysis. Thorax, 61, 140-5.

Siegel R, Ma J, Zou Z, et al (2014). Cancer statistics, 2014. CA Cancer J Clin, 64, 9-29.

Siegel R, Naishadham D, Jemal A (2013). Cancer statistics, 2013. CA Cancer J Clin, 63, 11-30.

Xiao H, Wang J, Liu Y, et al (2014). Relative influence of c-Kit expression and epidermal growth factor receptor gene amplification on survival in patients with non-small cell lung cancer. Oncol Lett, 8, 582-8.

Zhao F, Chen Y, Wu Q, et al (2014). Prognostic value of CD117 in cancer: a meta-analysis. Int J Clin Exp Pathol, 7, 1012-21. 